\title{
Energy from thin air
}

Advanced genomic-analysis techniques now suggest that microbial communities in cold, nutrient-poor Antarctic soils can acquire their energy from the oxidation of trace gases, rather than by photosynthesis. SEE LETTER P.400

\section{DON A. COWAN \& THULANI P. MAKHALANYANE}

I t might be surprising to some people that the Antarctic continent is not entirely covered by ice. Ice-free areas represent less than $0.4 \%$ of the continent's landmass ${ }^{1}$, and these regions are dominated by soils that range from water-saturated to hyper-arid. The extreme conditions preclude the growth of multicellular plants, apart from on the Antarctic Peninsula. As a result, most key ecosystem services in such cold, desert places are performed by bacteria. One of the most important energy-capture processes is light-driven photosynthesis in cyanobacteria, which fixes atmospheric carbon dioxide gas into carboncontaining molecules within the microbes ${ }^{2}$. On page 400 , Ji et al..$^{3}$ now report a little-known energy-capture mechanism that they propose occurs in extreme terrestrial environments in Antarctica in which cyanobacteria are uncommon or absent.

Photosynthetic microorganisms are usually rare in Antarctic soils. For example, cyanobacterial sequences represented less than $0.3 \%$ of the microbial sequences in the soil regions that the authors sampled. Although bacteria are thought to be the dominant drivers of biochemical cycling in these soils, and particularly in rock-associated habitats, the energy sources for the metabolic processes taking place in Antarctic soils had not been determined ${ }^{4}$.

Ji and colleagues used a combination of advanced genomic analysis, functional assays to monitor trace-gas oxidation, chemical analysis and energy-balance modelling to show that some extremely barren cold soils, such as the desert soils of the Antarctic periphery, support a previously unappreciated mode of aerobic energy acquisition in Antarctic microbes. The authors propose that these microbial communities are supported by a biological energy-capture process that relies on the oxidation of atmospheric hydrogen and carbon monoxide, termed trace gases because they represent less than $1 \%$ of the total amount of atmospheric gases.

The authors obtained DNA-sequence data sets from several samples of Antarctic soils at two locations (Robinson Ridge and Adams Flat), and used complex techniques to partly reassemble the draft genome sequences of key organisms in the soil. Some of these draft genome sequences belonged to two groups of little-known soil microorganisms, called WPS-2 and AD3, that have not been successfully cultured in a laboratory.

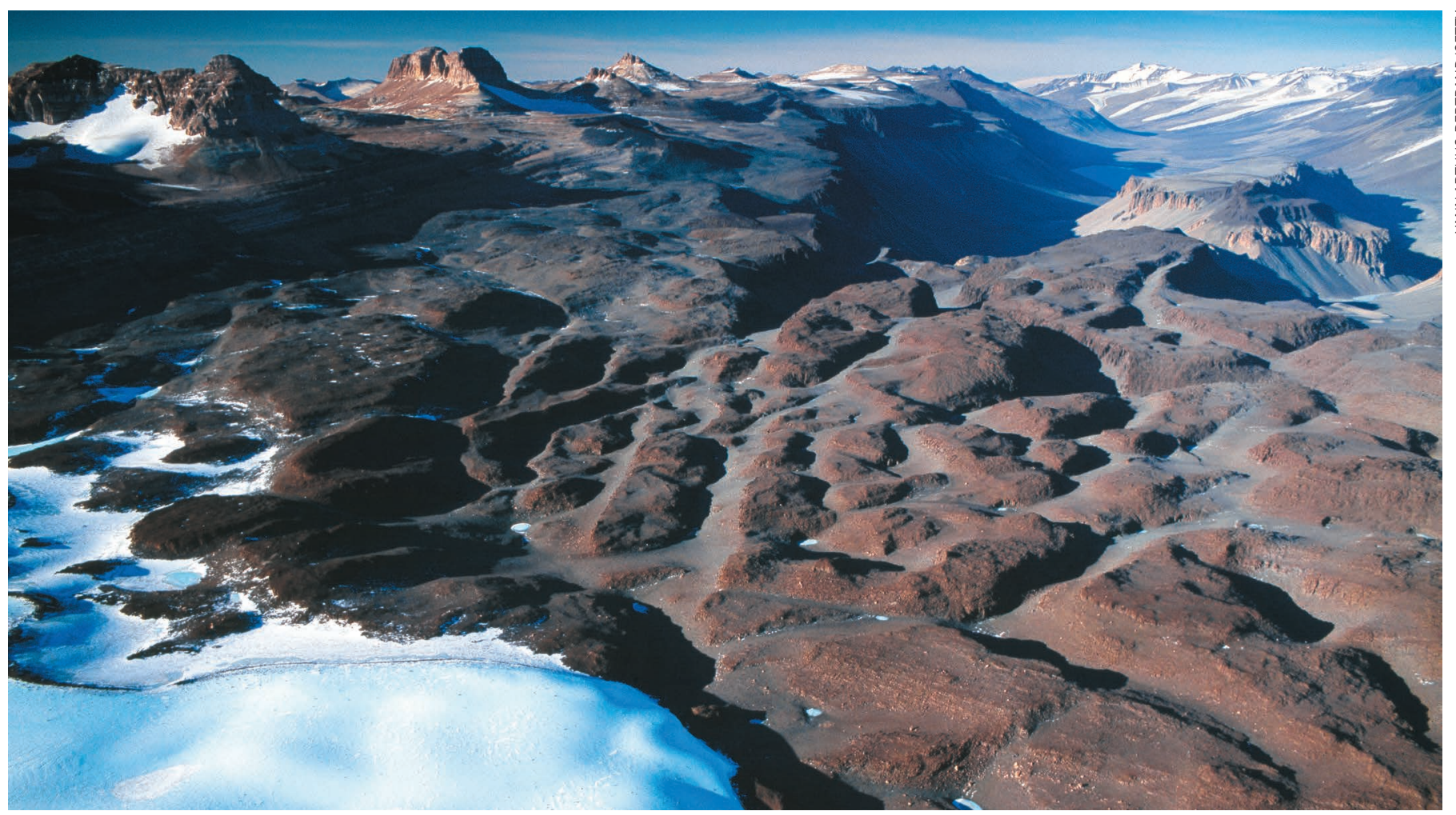

Figure 1 | Antarctic desert soils. Ji et al. ${ }^{3}$ report an analysis of microbial communities in ice-free desert regions of Antarctica called Robinson Ridge and Adams Flat. Their results suggest that some bacteria in these regions might obtain energy to fix carbon from the oxidation of hydrogen or carbon monoxide, rather than from photosynthesis. Genes associated with this proposed process are also found in publicly available DNA-sequence data sets of microbial samples obtained from arid soils of the McMurdo Dry Valleys in Antarctica (pictured). 
The authors analysed the metabolic potential of the dominant organisms in the samples by annotating the probable functions of the genes identified and noting putative metabolic pathways in the assembled draft genomes. Three particularly interesting types of gene were widespread. These encode enzymes that aid the oxidation of trace gases in energy-generating cellular pathways. The enzymes are: highaffinity hydrogenases, CoxLSMs (high-affinity carbon monoxide dehydrogenases) and type IE RuBisCOs ( $\mathrm{CO}_{2}$-fixation enzymes).

The proposed role of these key genes in tracegas oxidation was supported by biochemical analyses, in which the authors demonstrated that the same soil samples showed substantial and reproducible uptake of hydrogen and carbon monoxide, associated with increased $\mathrm{CO}_{2}$ fixation. This fixation process was not increased by light, indicating that photosynthetic processes were not involved.

However, caution might be called for when interpreting these findings. The genomicanalysis approach used by the authors can be biased towards organisms present in high abundance ${ }^{5}$. Even if cyanobacteria occur in low abundance, they might still make substantial contributions to key ecosystem services. The authors note that both photosynthesis and the trace-gas metabolism that they describe could co-occur in Antarctic soils, with the dominant process probably dependent on physicochemical factors such as water availability.

Ji et al. are careful to note that their evidence for this bioenergetics mechanism is restricted to some localized and specialized extreme Antarctic habitats. However, they propose that trace gases might be a more widespread energy source, having identified the key genes required for this metabolic process in several publicly available microbial DNA-sequence data sets obtained from sampling of an aridsoil region of Antarctica called the McMurdo Dry Valleys (Fig. 1). Yet why has this phenomenon not been observed previously in Antarctic soils? The probable reason is that no organisms showing this particular physiology have been cultured from Antarctic soils, and in desert soils in less-extreme environments, the dominant energy-acquisition process is cyanobacterial photosynthesis ${ }^{6}$.

The generation of energy through hydrogen and carbon monoxide metabolism has been observed before ${ }^{7}$. A wide range of microbes use hydrogen as a sole energy source to support $\mathrm{CO}_{2}$ fixation, although mostly under anaerobic (oxygen-free) conditions. Bacteria such as Geobacter sulfurreducens ${ }^{8}$ can scavenge atmospheric carbon monoxide as their sole energy source ${ }^{9}$, and carbon monoxide dehydrogenase genes are widespread in other strictly anaerobic extreme environments, such as deep sub-sea-floor sediments ${ }^{10}$.

There might be scepticism that the aerobic energy-capture process proposed by Ji and colleagues could suffice to maintain a microbial community. It seems instinctively implausible that the hydrogen and carbon monoxide levels are high enough in the cold, clear atmosphere of Antarctica to support life. Although the authors do not report the local atmospheric gas concentrations of the Robinson Ridge or Adams Flat sampling sites, they do present calculations showing that the oxidation of hydrogen and carbon monoxide can theoretically support the energy requirements of microbial populations in the Antarctic soil.

It is worth remembering that life in these environments is not exactly fast-paced, and cells are inactive for much of any year. The entire annual metabolic window - the period in which the organisms can be metabolically active - might be only a few hundred hours per year (compared to many thousands of hours for a moist soil in a temperate climate). Perhaps cell doubling takes days or months, rather than the minutes or hours needed in less-extreme environments.

Definitive proof of this proposed physiology will require the eventual isolation and laboratory culturing of these organisms, and the in vivo demonstration that such microbes can grow using hydrogen and carbon monoxide as energy sources. What is particularly exciting about Ji and colleagues' findings, especially for astrobiologists, is that it adds another dimension to our understanding of the survival and energy-sufficiency mechanisms of organisms living in places at the limits of where life is thought to be possible. The results might also stimulate further studies into the extent of hydrogen metabolism in other extreme environments.

Don A. Cowan and Thulani P. Makhalanyane are in the Department of Genetics, Centre for Microbial Ecology and Genomics,

University of Pretoria, Hatfield 0028, Pretoria, South Africa.

e-mail:don.cowan@up.ac.za

1. Bockheim, J. G. in Antarctic Terrestrial Microbiology (ed. Cowan, D. A.) Ch. 16 (Springer, 2014).

2. Cary, S. C., McDonald, I., Barrett, J. E. \& Cowan, D. A. Nature Microbiol. Rev. 8, 129-138 (2010).

3. Ji, M. et al. Nature 552, 400-403 (2017).

4. Chan, Y., Van Nostrand, J. D., Zhou, J., Pointing, S. B. \& Farrell, R. L. Proc. Natl Acad. Sci. USA 110 8990-8995 (2013).

5. Wu, Y.-W., Tang, Y.-H., Tringe, S. G., Simmons, B. A. \& Singer, S. W. Microbiome 2, 26 (2014).

6. Wood, S. A., Rueckert, A., Cowan, D. A. \& Cary, S. C. ISME J. 2, 308-320 (2008).

7. Conrad, R. Microbiol. Rev. 60, 609-640 (1996).

8. Geelhoed, J. S., Henstra, A. M. \& Stams, A. J. M. Appl. Microbiol. Biotechnol. 100, 997-1007 (2016).

9. Lalonde, I. \& Constant, P. Appl. Environ. Microbiol. 82, 1324-1333 (2016)

10. Hoshino, T. \& Inagaki, F. Lett. Appl. Microbiol. 64, 355-363 (2017).

This article was published online on 6 December 2017.

\title{
IMMUNOLOGY
}

\section{The origins of memory T cells}

\begin{abstract}
Memory T cells protect against previously encountered pathogens, but their origins are unclear. Two studies track DNA modifications over time and find that these cells arise from effector T cells. SEE ARTICLE P.362 \& LETTER P.404
\end{abstract}

\section{KYLA D. OMILUSIK \& ANANDA W. GOLDRATH}

V accination has substantially reduced illness and death from infectious disease by exploiting the ability of long-lived memory T cells to 'remember' a previous encounter with a specific microbe and mount a rapid response upon pathogen re-exposure. Understanding how immunological memory is established and maintained might provide insights that could enable improvements in vaccine design. In this issue, Akondy et al. ${ }^{1}$ (page 362) and Youngblood et al. ${ }^{2}$ (page 404) reveal the cell population that gives rise to memory $\mathrm{T}$ cells, and how the population of memory T cells evolves.

Naive T cells are those that haven't previously responded to a pathogen. When they recognize a pathogen, they rapidly divide and express molecules such as cytokine proteins that help to fight infection. These responding cells are called effector T cells (more specifically, a type of effector cell called a cytotoxic $\mathrm{T}$ cell) and they can migrate into inflamed tissues and kill infected cells ${ }^{3}$. Once the pathogen is eliminated, most effector cells die, but a small pool of long-lived memory cells remains that is poised to respond rapidly if reinfection occurs $^{3}$.

Which cells give rise to memory $\mathrm{T}$ cells has been extensively investigated. Two general possibilities (Fig. 1a, b) have been proposed: the cells either arise from a subset of the effector cells that escape death, or instead descend directly from naive T cells, which could, as early as their first cell division, give rise to cells with effector-T-cell or memory-T-cell potential $^{3}$. 